\title{
Real-time label-free biosensing with integrated planar waveguide ring resonators
}

Hans Sohlström, Kristinn B. Gylfason, Daniel Hill

Hans Sohlström, Kristinn B. Gylfason, Daniel Hill, "Real-time label-free biosensing with integrated planar waveguide ring resonators," Proc. SPIE 7719, Silicon Photonics and Photonic Integrated Circuits II, 77190B (17 May 2010); doi: 10.1117/12.855957

SPIE. Event: SPIE Photonics Europe, 2010, Brussels, Belgium 


\title{
Real-time label-free biosensing with integrated planar waveguide ring-resonators
}

\author{
Hans Sohlström*, Kristinn B. Gylfason, Daniel Hill \\ KTH-Royal Institute of Technology, School of Electrical Engineering, \\ Microsystem Technology Lab, Osquldas väg 10, SE-100 44 Stockholm, Sweden
}

\begin{abstract}
We review the use of planar integrated optical waveguide ring resonators for label free bio-sensing and present recent results from two European biosensor collaborations: SABIO and InTopSens. Planar waveguide ring resonators are attractive for label-free biosensing due to their small footprint, high $Q$-factors, and compatibility with on-chip optics and microfluidics. This enables integrated sensor arrays for compact labs-on-chip. One application of label-free sensor arrays is for point-of-care medical diagnostics. Bringing such powerful tools to the single medical practitioner is an important step towards personalized medicine, but requires addressing a number of issues: improving limit of detection, managing the influence of temperature, parallelization of the measurement for higher throughput and on-chip referencing, efficient light-coupling strategies to simplify alignment, and packaging of the optical chip and integration with microfluidics. From the SABIO project we report refractive index measurement and label-free biosensing in an 8-channel slotwaveguide ring resonator sensor array, within a compact cartridge with integrated microfluidics. The sensors show a volume sensing detection limit of $5 \times 10^{-6} \mathrm{RIU}$ and a surface sensing detection limit of $0.9 \mathrm{pg} / \mathrm{mm}^{2}$. From the InTopSens project we report early results on silicon-on-insulator racetrack resonators.
\end{abstract}

Keywords: slot waveguide, ring resonators, label-free, biosensing, lab-on-chip, light-coupling, packaging, microfluidics

\section{INTRODUCTION}

Refractive index sensing is widely used for real-time monitoring of chemical processes and, when used with separation techniques such as liquid chromatography or capillary electrophoresis, universal solute detection systems can by created. ${ }^{1}$

For biosensing, most established methods use special reporter molecules or particles, know as labels, to read out the measured quantity. Most often the labels are fluorescent or radioactive, but gold or magnetic nanoparticles are also used. Label-based sensors can measure down to the single molecule level, but they have a number of drawbacks. One limitation, often cited, is the time and cost of the labeling step. ${ }^{2}$ Another is the complexity that the label adds to the reaction under study, and thus an increased risk of misinterpretation. ${ }^{3}$ A more fundamental limitation is however that, since the labels are typically added in a secondary step, most labeled methods can only measure the static endpoint of a reaction and do not provide real-time information. This information can, however, be gained by label-free methods, which can give quantitative real-time information in physical units.

Since the refractive index of aqueous protein solutions is linear with protein density ${ }^{4}$, sensors measuring the refractive index close to a surface by using the evanescent field from a guided wave, can be used to measure the mass of protein binding on the surface. Refractive-index-based sensing methods can thus be used for label-free monitoring of biomolecular interactions on surfaces, as for example is done in the commercially successful surface plasmon resonance (SPR) based sensors. ${ }^{5}$

The advantages of scaling analytical chemical and biological instruments down to a single chip have been extensively explored. ${ }^{6}$ They include: automation of the analysis, increased mobility of the instrument, shorter response times, reduced manual sample handling, and a low cost per test. Thus, to bring the powerful tool of label-free sensing into the hands of a wider user base, there is a strong interest in integrating optical sensors in labs-on-chips that can be used at the point of care (POC), for example to the single medical practitioner or in a remote field situation.

*hans.sohlstrom@ee.kth.se; phone +46 8790 9041; fax +46 8100 858; www.ee.kth.se/mst

Silicon Photonics and Photonic Integrated Circuits II, edited by Giancarlo Cesare Righini,

Proc. of SPIE Vol. 7719, 77190B - (c) 2010 SPIE · CCC code: 0277-786X/10/\$18 · doi: 10.1117/12.855957 
Micro-fabrication technology allows efficient production of integrated optical devices at wafer scale. In this paper we will mainly discuss the application of integrated planar waveguide ring resonator. Due to its small footprint and ease of integration with other on-chip optical and fluidic functions, the ring resonator is a particularly interesting optical sensor for labs-on-chips.

In the following we will first review the use of optical ring resonators for biosensing and then give a summary of the use of such sensors in two European collaborative projects: SABIO and INTOPSENS.

\section{RING RESONATORS}

The theory of the ring resonator, side-coupled to a straight bus waveguide has been extensively studied ${ }^{7}$. Here we just conclude that if we start from a model according to Figure 1 and a make number of assumptions,

- all waveguides are unimodal per polarization orientation and do not couple between polarizations,

- all losses are incorporated in the attenuation constant of the individual modes,

- back reflections are negligible,

- transitions between modes are adiabatic,

- interaction between modes is negligible outside the coupler regions,

- only symmetric devices are considered,

- the coupling is lossless,

we can arrive at an expression for the power transmission in the forward direction through the bus waveguide.

$$
P_{T}=\left|D_{+}\right|^{2}=\frac{a^{2}+|\tau|^{2}-2 a|\tau| \cos (\theta+\psi)}{a^{2}+a^{2}|\tau|^{2}-2 a|\tau| \cos (\theta+\psi)}
$$

In this expression $a$ and $\theta$ are the loss and phase shift for a round trip in the ring, $\tau$ is the straight-through transmission of the coupler and $\psi$ is phase shift in the coupler. In Figure 2, $P_{\mathrm{T}}$ is plotted as a function of the round trip phase shift $\theta$ when $a \approx \tau$. In this case the transmission goes to zero in the dips. With this condition fulfilled, the dips become sharper when $a$ and $\tau$ are close to 1, i.e. when the losses are small. $\theta$ is proportional to the effective index of the ring guide and inversely proportional to the wavelength, so changing any of these would mean moving along this graph.

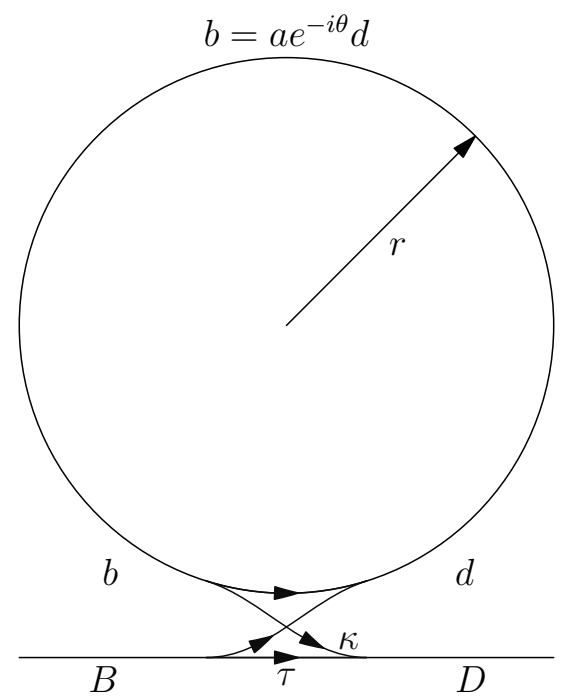

Figure 1. A waveguide ring resonator side coupled to a bus waveguide. $B, b, D$, and $d$ are the complex mode amplitudes. Ring modes are lower case and bus modes uppers case. $\kappa$ is the complex coupling coefficient and $\tau$ the complex transmission coefficient. Figure adapted from ${ }^{8}$. 


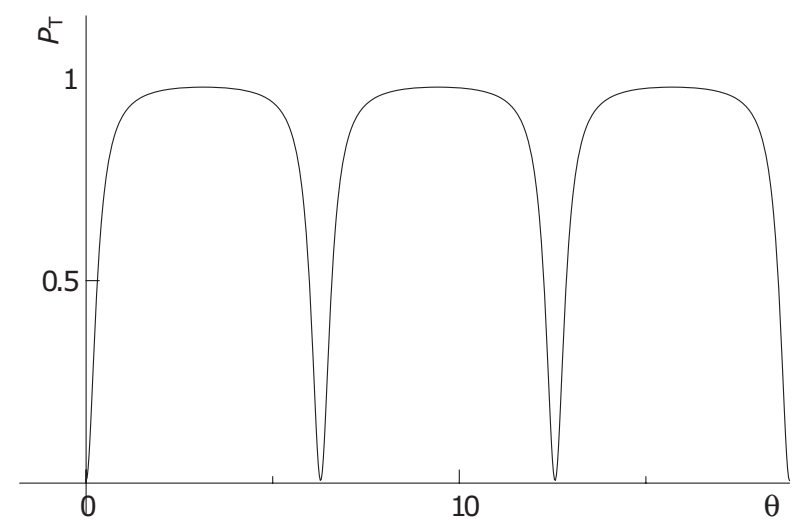

Figure 2. The transmitted power $P_{\mathrm{T}}$ of a side coupled ring resonator, for $a=0.87$ and $\tau=0.85$, as functions of the round trip phase shift $\theta$ and ignoring dispersion. Figure adapted from ${ }^{8}$.

To achieve a good resolution in terms of refractive index of the sample we must strive for

- a large change in the effective index of the guide for a given change in sample index or, expressed in another way, a large sensitivity in terms of resonance wavelength shift per refractive index unit (RIU),

- low losses in the resonator ring to achieve sharp dips in the ring transmission characteristics

- an efficient method to determine the changes in the dip position,

- suppression of changes in the effective index from interferences, such as the temperature.

In the following we will se that all these factors are important to improve the detection limit.

\section{RING RESONATORS FOR BIOSENSING}

Much of the early work on planar optical waveguides for label-free sensing was performed at ETH Zürich, Switzerland, by Lukosz and Tiefenthaler in the 1980s and early 1990s. Most of this was done on sol-gel formed slab waveguides, with optical confinement only in the out-of-plane dimension. Nevertheless, much of the early theoretical sensitivity analysis of such waveguide sensors ${ }^{9,10,11}$ remains a useful guideline. For reading out changes in the effective index of the waveguide, Lukosz and Tiefenthaler used surface gratings embossed into the waveguiding layers. ${ }^{12}$

Some other early contributions were made at the MESA Research Institute, University of Twente, the Netherlands. There, more complex devices, such as integrated Mach-Zehnder ${ }^{13}$ and TE-TM difference interferometers ${ }^{14}$ with integrated photodetectors, were developed. However, to achieve a low limit of detection, Mach-Zehnder interferometers need a long sensing arm (usually a few $\mathrm{mm}$ ).

In contrast to Mach-Zehnder waveguide interferometers, the degree of interaction of light and sample in ring resonators is not limited by the physical length of the sensing waveguide, but rather by the number of oscillations of light in the resonator, characterised by the quality factor $Q$.

In 1997, Sohlström and Öberg at IMC AB (currently Acreo AB), Kista, Sweden reported the use of planar ring resonators for measuring refractive index. ${ }^{15}$ Their device operated at a wavelength of $1550 \mathrm{~nm}$ and was made from $\mathrm{Si}_{3} \mathrm{~N}_{4}$ strip waveguides on silicon oxide. It included an integrated splitter for splitting light to a sensing arm and a reference arm, each coupled to ring resonators of $1 \mathrm{~mm}$ radius. The waveguides had a thin core with $140 \mathrm{~nm}$ thickness to achieve a high refractive index sensitivity by pushing optical energy out into the sample region. Based on experiments with repeated injections of $1 \%$ sucrose solutions and using a curve-fitting approach to efficiently detect the resonance wavelength, the authors estimated a volume refractive index detection limit of $5 \times 10^{-6}$ RIU.

In 2002, Krikouv et al. at Twente University demonstrated a similar sensor based on a $\mathrm{Si}_{3} \mathrm{~N}_{4}$ disk resonator with a $15 \mu \mathrm{m}$ radius. ${ }^{16}$ Since the devices utilized a vertical coupling scheme for better control of the critical coupling distance between ring and bus waveguides, they could be fabricated by standard UV lithography. The small disks displayed 3 radial 
whispering gallery (WG) modes, and thus sensing required proper interpretation of the multi-mode transmission spectrum. The devices showed a volume refractive index sensitivity of $23 \mathrm{~nm} / \mathrm{RIU}$ and a detection limit of $10^{-4}$ RIU.

In 2003, Chao and Guo at the University of Michigan, Ann Arbor, showed volume refractive index sensing with an imprinted polystyrene (PS) ring sensor on a silicon dioxide cladding. ${ }^{17}$ In a follow-up paper in $2006{ }^{18}$, these devices were shown to have a detection limit of $5 \times 10^{-5}$ RIU.

In early 2006, two papers were published on biomolecule surface sensing with ring resonators. Chao and Guo showed binding of streptavidin to a biotin coated surface of a PS ring resonator. ${ }^{18}$ The authors estimated a surface mass density detection limit of $250 \mathrm{pg} / \mathrm{mm}^{2}$. Yalcin et al. at Boston University and Nomadics Inc. also worked with avidin-biotin binding, but on a Hydex glass ring. ${ }^{19}$ The authors did not estimate the surface mass detection limit, but the rings showed a volume refractive index detection limit of $1.8 \times 10^{-5}$ RIU.

At about the same time, Ksendzov and Lin at the California Institute of Technology also published on surface sensing of biomolecules. ${ }^{20}$ They used a $\mathrm{Si}_{\mathrm{X}} \mathrm{N}_{\mathrm{Y}}$ ring of $2 \mathrm{~mm}$ radius on a $\mathrm{SiO}_{2}$ cladding excited with a $\mathrm{HeNe}$ laser at $633 \mathrm{~nm}$. The authors estimated an avidin volume mass density detection limit of $6.8 \mathrm{ng} / \mathrm{ml}$ for their setup, but since the mass transport properties of the flow system have a large influence on this figure, comparison with other devices is difficult.

Because of the high refractive index contrast of silicon on silicon oxide as compared to for example silicon nitride on oxide, silicon waveguides allows smaller rings without reducing $Q$ by bending loss. Also the electric field at the silicon surface is high, yielding high sensitivity for surface sensing. In 2007, De Vos et al. at Ghent University presented biosensing with silicon rings of only $5 \mu \mathrm{m}$ radius ${ }^{21}$ and showed a detection limit of $17 \mathrm{pg} / \mathrm{mm}^{2}$ in a follow-up paper. ${ }^{22}$

In 2004 Almeida et al. at Cornell University demonstrated slot waveguides ${ }^{23,24}$, consisting of two rails of a high index material separated by a low index slot region of sub-wavelength width. With proper design, this double core structure acts as one waveguide and supports only the lowest order transverse electric (TE) and transverse magnetic (TM) modes, with a considerable fraction of the power of the TE mode propagating in the low index slot. This is exemplified in Figure 3 from $^{25}$. With a liquid sample extending into the slot, this may give a higher refractive index sensitivity than with conventional strip guides. In 2007, Barrios et al. demonstrated the use of $\mathrm{Si}_{3} \mathrm{~N}_{4}$ slot-waveguides for bio-sensing, achieving a sensitivity of $212 \mathrm{~nm} /$ refractive index units (RIU) ${ }^{26}$ This was the first result of the SABIO project, which will be further discussed below.

Also, guides with several slots have been demonstrated. ${ }^{27}$ They theoretically have an even higher refractive index sensitivity, but this comes at the cost of an increased loss due to an overall greater side surface area contributing to the light scattering.
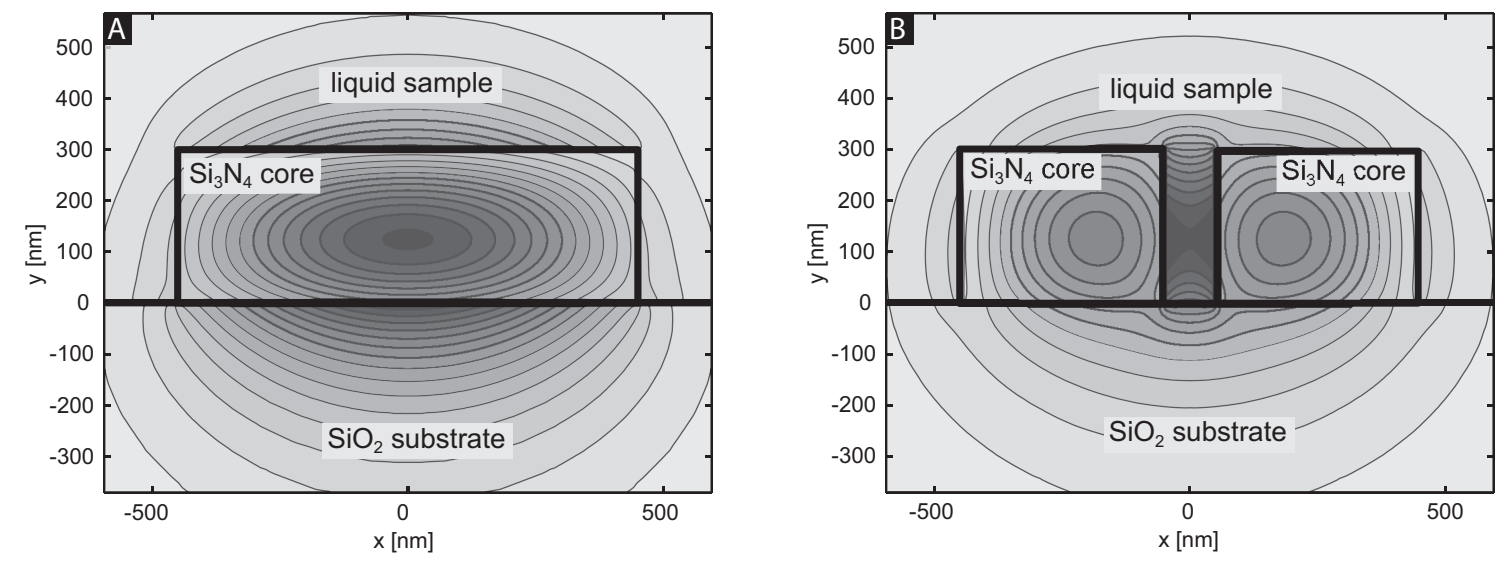

Figure 3. Contour plots of the simulated average optical power density flowing through two different waveguide crosssections at a wavelength of $1310 \mathrm{~nm}$ : (A) In the TM mode used for sensing with a strip waveguide, most of the optical power propagates in the waveguide core. (B) In the slot waveguide TE mode, however, up to half of the power propagates in the liquid sample, thus yielding a higher sensitivity to refractive index changes of the sample. The gray scales of the two images have the same normalisation. Figure from ${ }^{25}$. 
In contrast to Mach-Zehnder interferometers, the ring resonator does not include a reference arm. However, an on-chip reference is useful for compensation of temperature and other interfering inputs. Furthermore, for POC applications, the simultaneous detection of several surface reactions is useful. Thus, there is an interest in on-chip sensor arrays.

The first steps towards integrated ring resonator sensor arrays were taken by Ramachandran et al. at Nomadics Inc. in 2007 with 5 rings integrated on a single chip. ${ }^{28}$ However, instead of an integrated optical approach, individual input and output fibres were coupled to each ring and light splitting was handled off-chip.

In 2008, Hu et al. at the Massachusetts Institute of Technology, presented a number of important advances. ${ }^{29}$ They showed single mode disk resonators of $20 \mu \mathrm{m}$ radius in chalcogenide $\left(\mathrm{Ge}_{17} \mathrm{Sb}_{12} \mathrm{~S}_{71}\right)$ glass. The resonators showed a $Q$ of $2 \times 10^{5}$ in polymer and $2 \times 10^{4}$ in water. The high $Q$ in combination with a high refractive index sensitivity of $182 \mathrm{~nm} / \mathrm{RIU}$, yielded a refractive index limit of detection as low as $8 \times 10^{-7}$. A new type coupler relaxes the tolerance on the coupling distance, and hence the horizontally coupled disks can be fabricated by normal UV lithography in a single lithography step.

Claes et al. at Ghent University showed sensing with silicon slot waveguide rings in $2009 .^{30}$ The rings have a radius of only $5 \mu \mathrm{m}$, a slot width of $104 \mathrm{~nm}$, and are fabricated by Deep-UV $(193 \mathrm{~nm})$ lithography. The devices show a large refractive index sensitivity of $298 \mathrm{~nm} / \mathrm{RIU}$, but relatively high bending losses limit the $Q$ of the device to 330, thus yielding a detection limit of $4 \times 10^{-5}$. The authors also performed label-free biosensing, but do not give a surface mass density detection limit. This was an early result from the InTopSens project, which will be further discussed below.

In late 2009 and early 2010, Washburn et al. at the University of Illinois at Urbana-Champeign demonstrated biosensing measurements in bovine serum ${ }^{31}$ and a multiplex assay of cancer biomarkers. ${ }^{32}$ The sensors are SOI rings of $15 \mu \mathrm{m}$ radius and light is coupled in and out of the chip with surface grating couplers.

Recently, specially manufactured, non-planar, sphere- and toroid-resonators with very high $Q$-values have been reported to detect single viruses ${ }^{33}$ and even single proteins ${ }^{34}$, even though the physical explanation given for the latter report has recently been disputed. ${ }^{35}$ These reports show that the fundamental detection limit of ring resonator sensors lies well beyond what is needed for many applications. However, the special re-flow or melting techniques applied to obtain the high quality factors of these devices are not directly compatible with standard wafer scale fabrication.

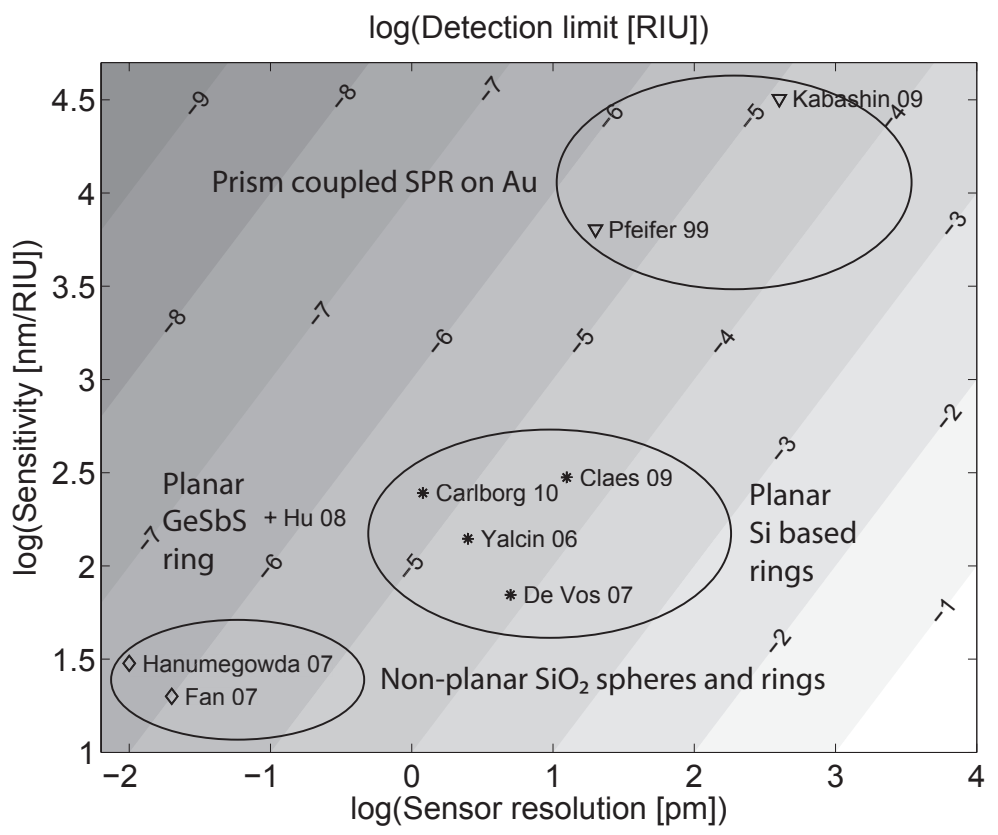

Figure 4. Detection limit analysis, from ${ }^{8}$. The $x$-axis is the log of the wavelength resolution and the $y$-axis is the log of the device sensitivity in terms of wavelength shift per RIU. The grayscale then represents the minimum detectable refractive index change. Examples included are Hu et al. ${ }^{29}$, Haneumegowda et al. ${ }^{36}$, Fan et al. ${ }^{37}$, Carlborg et al. ${ }^{25}$ (the SABIO device), Claes et al. ${ }^{30}$ (an InTopSens prototype), Yalcin et al. ${ }^{19}$, De Vos et al. ${ }^{21}$, Pfeifer ${ }^{38}$ and Kabashin et al. ${ }^{39}$. 
Some of these examples are included in the analysis in Figure $4^{8}$. It is a contour plot of a figure of merit formed as is the ratio of the sensitivity in terms of $\mathrm{nm}$ resonance shift per RIU (bigger is better) and the sensor system wavelength resolution (smaller is better), with some reported experimental values superimposed on the plot.

Three classes of devices can be distinguished. In the top right corner we find the high sensitivity, low $Q$ devices. Surface Plasmon resonance sensors are in general of this class. Since they have a very large index contrast at the metal/dielectric interface, high electric field strengths are obtainable in the dielectric sample. However, since the plasmon propagation is lossy, the resonance is not sharp.

On the opposite end of the scale, in the bottom left corner, we find non-planar toroid ring and spherical resonators, made by reflowing glass. These have most of the electric field in the core, and in general a low sensitivity. However, losses in the dielectric resonators are extremely small, and due to the reflow, surface roughness is very small and scattering low.

In the centre, we find a cluster of planar waveguide ring resonators. These devices have lower sensitivities than the plasmon based devices, because of the lower index contrast, but since they are made only of dielectrics they do not suffer the electron scattering losses. These devices are theoretically lossless except for bending losses. In reality, however, photon scattering from the roughness of the lithographically defined sidewalls limit the quality factors.

The slot waveguides allow higher sensitivity and thus come higher up in Figure 4. The guide is still singlemode and the modes are theory lossless except for possible bending losses. But there is a catch, if the extra side surfaces in the slot guide are created by lithography and etching, the number of rough surfaces has also increased. The penalty is increased losses, a lower $Q$, and in turn increased noise, i.e. a drift to the right in the figure.

White and Fan ${ }^{40}$ recently analysed the factors influencing the detection limit of optical resonant refractive index sensors. Their analysis shows that low $Q$ sensors are typically limited by amplitude noise and spectral resolution, while high $Q$ sensors are limited in performance by temperature stabilisation. An increase in sensor sensitivity will thus not yield an improvement in detection limit, unless these limitations are first addressed.

As far as we know, ring resonator based sensors are not yet commercially available, but there are some very recent indicators towards commercialization. Washburn et al. recently published two papers on label-free sensing of cancer biomarkers. ${ }^{31,32}$ The resonator array substrates and the instrumentation for analyzing the microring resonance frequencies were acquired from Genalyte, Inc. (San Diego, CA, USA), but so far Genelyte doesn't offer the system for sale.

\section{THE SABIO APPROACH}

\subsection{The SABIO cartridge}

To demonstrate the possibility of using a slot ring resonator device in a point-of-care setting, the SABIO measurement cartridge, Figure 5, was designed for non-critical alignment in a reader instrument. An on-chip fluidic channel network layer is included to transport the samples to the sensors. Alignment pins and precision dicing of the chip minimize the position inaccuracy when mounting the cartridge in the instrument.

Light is coupled into the cartridge from above, using an alignment-tolerant grating coupler. ${ }^{41}$ The light is then distributed to the different sensors on the chip and coupled out through the edge of the chip in a way that allows automatic alignment to a photo-diode array. ${ }^{42}$ The fluidic layer is on top of the optics. A full description of the cartridge design appears in ${ }^{25}$, while here we will summarize only the key points.

The integration of sample handling on-chip is important to reduce size and simplify the analysis in compact analysis platforms. Manual sample and reagent handling should be replaced by a microfluidic network that allows handling of sub-microlitre volumes.

The most commonly used material for such microfluidic sample handling networks is poly(dimethylsiloxane), PDMS, a bio-compatible, transparent, rubber-like polymer in which features can be replicated down to nanometer dimensions using soft lithography. ${ }^{43}$ To bond the PDMS to an optical chip layer, the polymer surface can be activated in an oxygen plasma to expose hydroxyl groups that can form covalent bonds when brought in contact with silicon or glass substrates. ${ }^{44}$ However, the properties of PDMS make it unsuitable as a protective packaging material, in particular it deforms easily under pressure. A hard outer shell is therefore typically needed. 


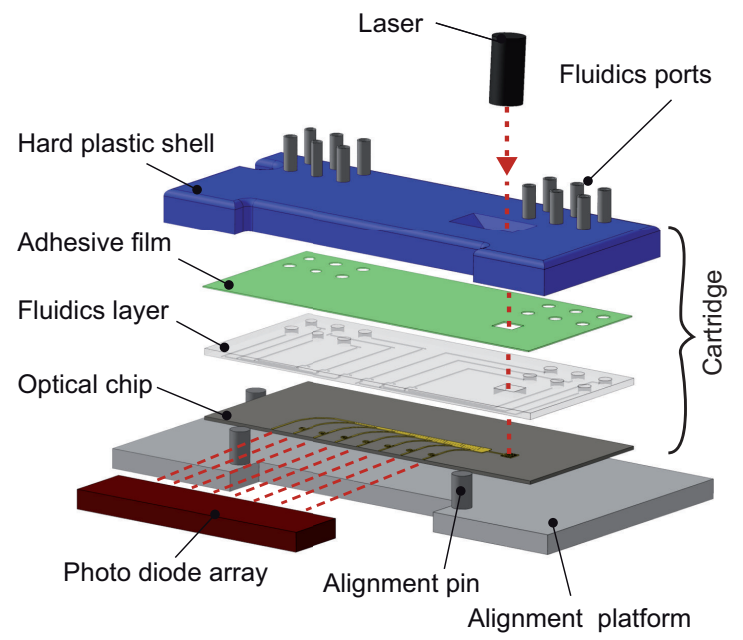

Figure 5. The SABIO cartridge.

Unfortunately, the rubber-like surface of PDMS adheres poorly to many materials used in packaging. Therefore, the most common packaging solution for labs-on-chips based on PDMS is to clamp the soft polymer layer between the sensor chip and a hard plastic shell. This configuration, however, can leak at high pressure. ${ }^{45}$ There is also a risk that shallow channels in the soft material will be blocked if the package is unevenly clamped.

In the SABIO cartridge, the problem with the adhesion to the packaging material was solved by using a dual surfaceenergy adhesive film, where one side of the film adheres to the PDMS and the other to a hard plastic shell used to package the chip.The SABIO microfluidic distribution layer is manufactured in PDMS by standard soft lithography methods. Each of the six sensors is addressable by a separate fluidic channel, with cross-sections of $200 \times 20 \mu \mathrm{m}^{2}$ at the widest section over the transducer. These dimensions assure that advection dominates over diffusion, allowing accurate measurement of fast binding dynamics. When the PDMS structure is brought in contact with the clean oxide surface of the optics chip, a leak-tight bond is formed.

A hard PMMA shell protects both the soft PDMS layer and the optics chip and provides an interface for the fluidic inputs and outputs. To ensure alignment of the optical chip to the instrument, cutouts for alignment pins are milled into two edges of the PMMA to allow physical contact between the pins and the precision cut silicon chip. Steel tubing for interfacing with the fluidic network is glued into the through holes.

The adhesive film (5302A, Nitto Denko, Japan) that is used to mount the hard shell on the PDMS is cut to the dimensions of the PDMS layer. The film is then sandwiched between the shell and the microfluidic layer. The resulting bond has been tested by pressurising the finalised cartridge with up to 1 bar of air pressure without leakage.

\subsection{SABIO optical chip design}

Parallel sensor operation not only yields higher throughput by multiple analyses of one sample, or simultaneous analyses of multiple samples, but it can also provide reference channels for drift compensation and control experiments. Such reference measurements are particularly important for automated labs-on-chips and chips without temperature stabilisation. In our case the chip is designed with 6 measurement channels and two reference channels.

In this work, the optical chip consists of a silicon substrate, with the integrated optical components etched into a silicon nitride thin-film, embedded in a silicon dioxide cladding on its surface. Since silicon nitride is nearly impervious to diffusion of moisture and sodium ions, it maintains a stable refractive index when operating in biological liquids. Additionally, silicon oxide and nitride have a relatively high refractive index contrast, thus permitting close spacing of integrated optical components, by allowing small bend radii without excessive bending losses. In comparison to the even higher index contrast in the silicon/silicon oxide system, it eases the problems from loss due to surface roughness. 


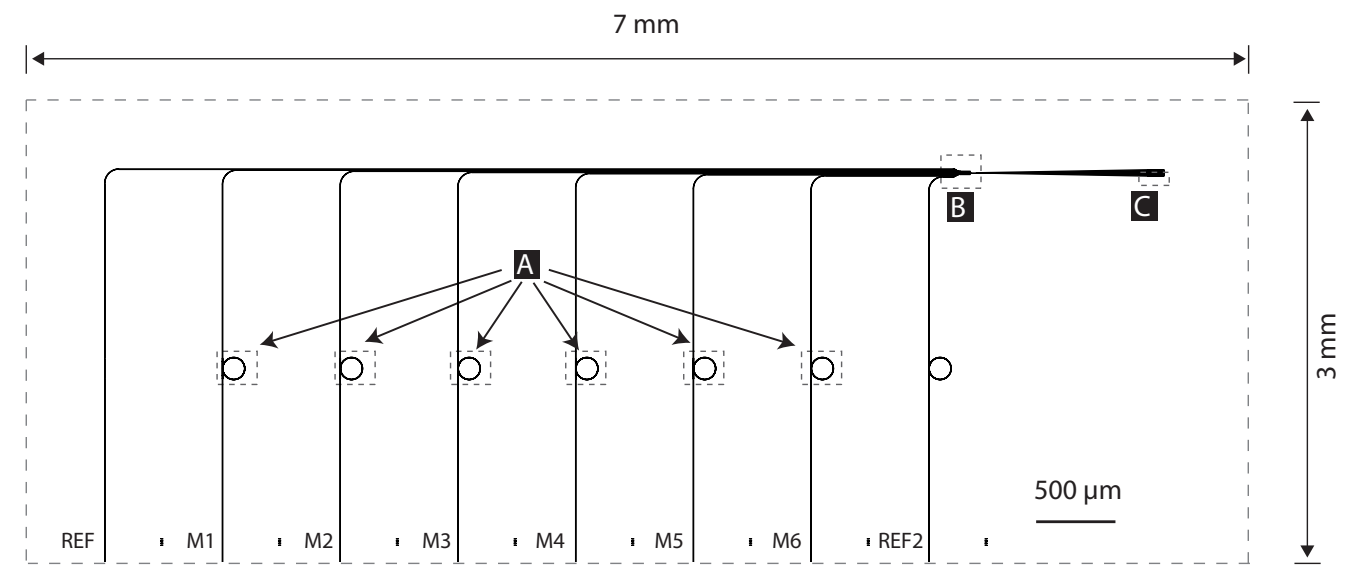

Figure 6. The optical chip: Light is injected at the surface grating coupler (C) and split, by the multi-mode interference splitter (B), to the six sensing channels M1-M6 and the two reference channels REF1 and REF2.

Figure 6 shows the optical circuit, occupying a chip area of $3 \times 7 \mathrm{~mm}^{2}$. Light enters from above via the surface grating coupler $(\mathrm{C})$ designed to give relaxed tolerances on the positioning while maintaining a high-efficiency coupling. ${ }^{41}$ The coupler is through-etched and thus does not complicate the fabrication. The propagating light is then split, by a multimode interference splitter (B), into eight channels: measurement channels M1 to M6 containing the sensing sites where openings have been etched in the silicon dioxide top cladding to allow liquid sample access down to the slot-waveguide ring resonators (A) and two reference channels. ${ }^{42}$ More information on the design can be found in ${ }^{25}$ and ${ }^{46}$.

In the chip design, channel-to slot mode converters are used for conversion between the two waveguide types before and after the ring resonator coupling regions, where the bus slot-waveguides have rail widths of $400 \mathrm{~nm}$ and a slot width of $200 \mathrm{~nm}$, Figure 7. The coupling gap is $350 \mathrm{~nm}$. In the sensing ring, asymmetric slot-waveguides with the inner rail widened to $550 \mathrm{~nm}$ are used for high optical confinement in the slot and low bending loss.

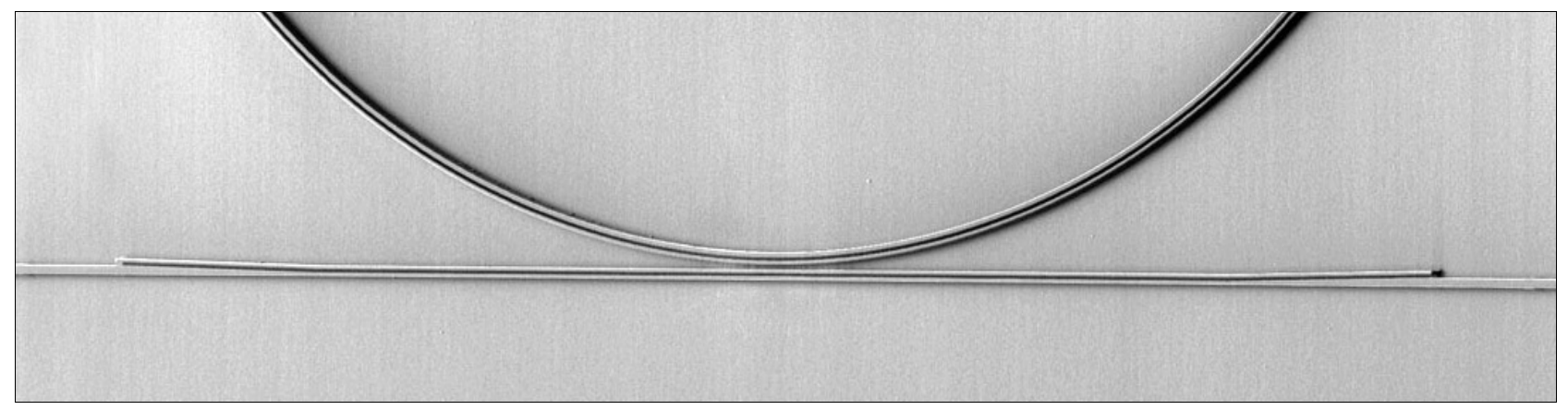

Figure 7. SEM image of the bus waveguide with the two channel-to slot mode converters and part of the ring on the SABIO chip.

The choice of laser wavelength is a trade-off between a narrow slot-width at short wavelengths, that may limit analyte access, and light absorption in water at longer wavelengths, that limits the resonator $Q$ factor. Our choice of the standard telecommunication wavelength of $1310 \mathrm{~nm}$ is a compromise ensuring that a wide selection of sources is available.

\subsection{SABIO optical chip fabrication}

For the fabrication, a $3.26 \mathrm{~mm}$ thick bottom cladding is first grown by thermal oxidation of the silicon wafer. A $300 \mathrm{~nm}$ silicon nitride device layer is then deposited by low pressure chemical vapour deposition (LPCVD). Next, the device layer is patterned by electron beam lithography and dry etching. A negative electron beam resist (ma-N 2403, Micro Resist Technology, Germany) is well suited for patterning narrow optical waveguides, since only a small fraction of the surface needs to be exposed. For mass production, standard deep ultra-violet lithography could be used. The patterned 
waveguides are then covered by a $530 \mathrm{~nm}$ thick top cladding layer of silicon dioxide deposited by tetraethyl orthosilicate (TEOS) based LPCVD and openings down to the 6 transducer sites are patterned by optical lithography and wet etching in buffered hydrofluoric acid. The thicknesses of the top and bottom oxide cladding layers are chosen for maximum coupling efficiency of the input grating coupler.

\subsection{SABIO temperature compensation}

To present a useful alternative to current technology, novel sensors need to achieve a detection limit of the order of $10^{-6}$ refractive index units (RIU) or better. Considering the fact that commonly used waveguide materials, and the liquid solvents of interest have thermo-optic coefficients of magnitudes $10^{-5}-10^{-4} \mathrm{RIU} / \mathrm{K}$, it is clear that minimizing temperature interference is essential. An uncompensated sensor requires temperature stabilization to of $10-100 \mathrm{mK}$ to reach the required performance.

Three approaches have been used for thermal noise reduction: active temperature control, athermal waveguide design, and temperature drift compensation by on chip referencing.

The first approach, active control of system temperature, can be implemented with external Peltier heat pumps. Temperature stability in the $10 \mathrm{mK}$ range is feasible. However, the required components limit the cost benefits gained by employing silicon micro-fabrication. Also they impede portability. We note, however, that for measuring chemical reaction rates, some degree of absolute temperature control is required, since rate constants are temperature dependent.

In the second approach, athermal waveguide sensors can be created by taking advantage of the different polarity of the thermo-optic coefficients of liquid samples and solid waveguide materials. For example, water has a negative thermooptic coefficient of $-10^{-4} \mathrm{RIU} / \mathrm{K}^{47}$, while silicon nitride and oxide have a positive value of $10^{-5} \mathrm{RIU} / \mathrm{K} .^{48}$ By balancing the fraction of light propagating in each material, the temperature dependence of the waveguide effective index can be eliminated. Athermal planar waveguide wavelength filters with solid polymer top cladding have been studied for almost two decades ${ }^{49}$, and also recently suggested for non-integrated sensors by Suter et al. ${ }^{50}$

In the third approach, temperature drift compensation by on-chip references, multiple identical sensors in good thermal contact with each other are integrated on the same substrate. With a fluidic system allowing injection of the sample of interest to one sensor, and a reference sample to another, differential measurements can be made. Such designs are solvent independent and tolerant to chip-to-chip fabrication variation.

In the SABIO device we use the design freedom achieved with the slot waveguide to achieve sensors with reduced temperature sensitivity and then use on-chip referencing to overcome the residual temperature sensitivity. This is further described in ${ }^{46}$.

\subsection{SABIO measurements and results}

A tunable laser was stepped over a range containing a least one ring resonator dip in each channel. The wavelength positions of the dips in the ring characteristic were found by fitting a combination of a simplified Lorentzian form of the ring behavior given above and a representation of an interfering Fabryt-Perot behavior from other components in the chip to the measured data. ${ }^{46}$ This pushes down the wavelength noise significantly below the laser tuning step. The volume refractive index sensitivity and limit of detection of the sensor chip was determined by injecting a dilution series of ethanol and methanol plugs into a running buffer of deionised (DI) water. Table 1 lists the refractive index shifts for the concentrations used. ${ }^{51}$ Real-time multiplex operation was demonstrated by concurrently measuring ethanol and methanol dilutions in channels M1 and M2, respectively, while channels M3 and M4 contained still standing DI water, to serve as references to monitor any drift of the DI water baseline. In a second part of the experiment, the roles of M1 and M2 were interchanged to quantify the sensitivity difference between two sensors on the same chip. A full description of the experiments is given in ${ }^{25}$. 
Table 1. Refractive indices of the solutions

\begin{tabular}{cc}
\multicolumn{2}{c}{ Ethanol } \\
\hline Mass percentage & Refractive index shift \\
\hline 5.97 & 0.0037 \\
3.98 & 0.0024 \\
1.99 & 0.0012 \\
0.994 & 0.006 \\
0.500 & 0.003 \\
\multicolumn{2}{c}{ Methanol } \\
\hline Mass percentage & Refractive index shift \\
\hline 20.1 & 0.0051 \\
9.82 & 0.0036 \\
5.00 & 0.0023 \\
\end{tabular}

Figure 8 (A) shows the resonance wavelengths of transducers M1-M4, as functions of time, during injections in M1 and M2. As expected, the resonance wavelengths of M1 and M2 are shifted during injection of the higher refractive index samples and then return to baseline as the flow returns to DI water. Furthermore, the magnitude of the shift correlates with the concentration injected.

A slight upward drift of the M1 and M2 baselines can be seen during the initial repeated high concentration injections. We attribute this drift to the known organic solvent absorption of PDMS. ${ }^{52}$

To find the refractive index sensitivity, first the channels M1 and M2 are compensated for the drift observed in M4. The compensated time trace of the resonance wavelength shift of M1 and M2, is shown in Figure 8 (B). The remaining baseline red shift of $50 \mathrm{pm}$ in 100 minutes is thus due to solvent absorption. The volume refractive index sensitivity is then determined by plotting the measured resonance wavelength shift as a function of the refractive index shift of the solutions injected. This gives an index sensitivity of $S_{\mathrm{n}}=246 \mathrm{~nm} / \mathrm{RIU}$.

The inset in Figure 8 (B) shows the baseline noise. Following the convention of using 3 standard deviations $\sigma$ of the total system noise as a measure of the sensor resolution, we get:

$$
R=3 \sigma=1.2 \mathrm{pm}
$$

The volume refractive index detection limit is then given by $L_{n}=R / S_{n}$ and thus

$$
L_{n}=5 \times 10^{-6} \mathrm{RIU}
$$
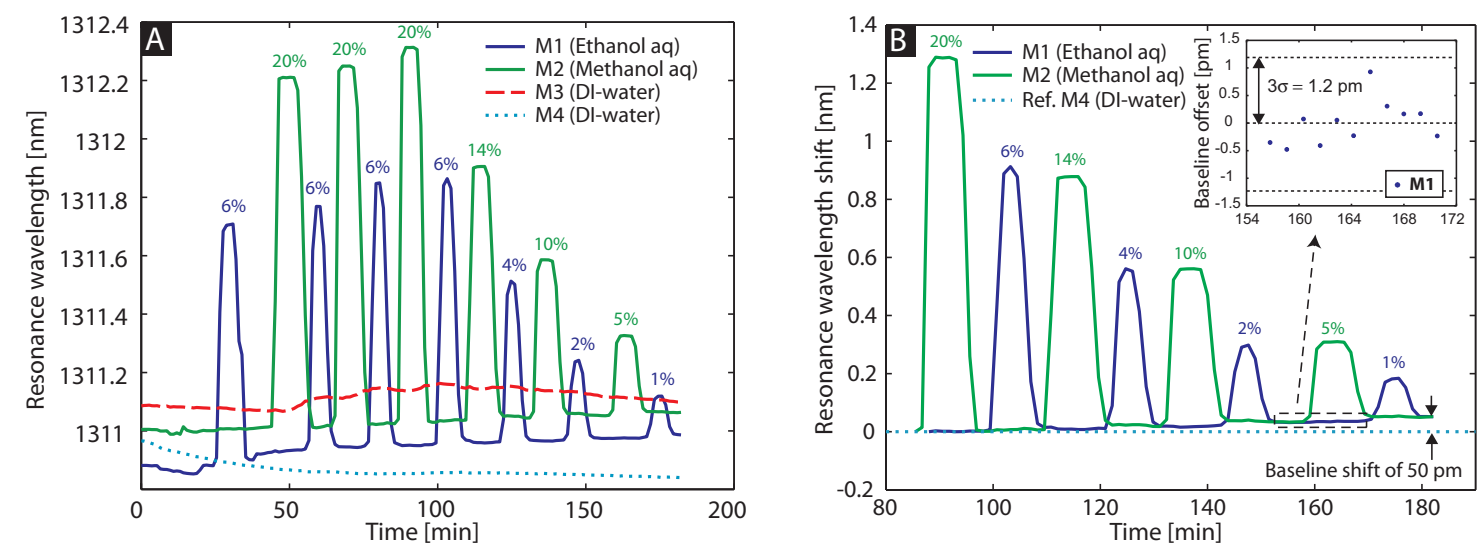

Figure 8. (A) Resonance wavelengths of transducers M1-M4, during ethanol and methanol injections into a running buffer of DI water in channel M1 and M2, respectively. Channels M3 and M4 contain still standing DI water for reference.

(B) The resonance wavelength shift of transducers M1 and M2 during ethanol and methanol injections, compensated for temperature drift by using M4 (DI-water) as reference. Graphs from ${ }^{25}$. 

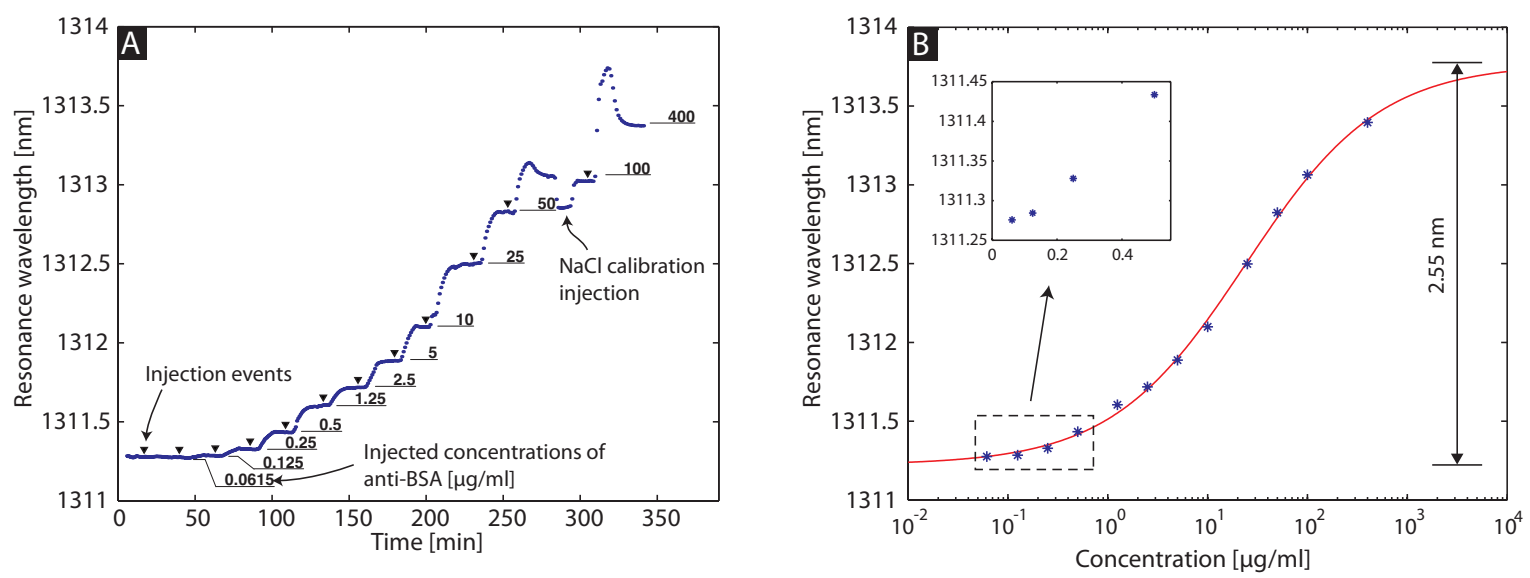

Figure 9. (A) The time trace of increasing concentrations of anti-BSA binding to the glutaraldehyde coated transducer. Injection times are marked with filled triangles and the injected concentration noted at the plateau level. (B) The resonance wavelength as a function of injected anti-BSA concentration. The solid curve is a fitted sigmoid model. The inset shows the first four points on a linear concentration scale. Graphs from ${ }^{25}$.

We also studied the surface mass sensing performance by measuring the binding of anti-BSA to a surface selectively activated by a layer of the molecular linker glutaraldehyde. Increasing concentrations of anti-BSA were then injected in a running buffer of phosphate buffered saline (PBS) and the resonance shift monitored until saturation.

Figure 9 (A) shows the resonance wavelength, as function of time, during binding of anti-BSA to the waveguide surface. Increasing concentrations, from $0.061 \mu \mathrm{g} / \mathrm{ml}$ to $400 \mu \mathrm{g} / \mathrm{ml}$, are injected. The time of each injection is indicated in the plot by a filled triangle. At a concentration of $0.125 \mu \mathrm{g} / \mathrm{ml}$, a shift is clearly visible and at concentrations exceeding $50 \mu \mathrm{g} / \mathrm{ml}$ we observe removal of non-covalently bound material after each injection, indicating that the surface is saturating.

The resonance wavelength as a function of anti-BSA concentration, shown in Figure 9 (B), fits well to a typical sigmoid curve for binding site limited reactions, and we can estimate the shift in resonance wavelength at saturation to $\Delta \lambda=2.55$ $\mathrm{nm}$. The sensitivity for surface mass detection is given by $S_{\mathrm{m}}=\Delta \lambda / \sigma_{\mathrm{p}}$, where $\sigma_{\mathrm{p}}$ is the surface density of a molecular monolayer. The surface density of a monolayer of anti-BSA measured using dual polarisation interferometry with the Farfield AnaLight 4D system was $\sigma_{\mathrm{p}}=2.0 \mathrm{ng} / \mathrm{mm}^{2}$. Using the value of the resonance shift from the experiment, we can thus calculate a mass sensitivity of $S_{\mathrm{m}}=1.3 \mathrm{~nm} /\left(\mathrm{ng} / \mathrm{mm}^{2}\right)$. Since the surface mass detection limit is given by $L_{\mathrm{m}}=R / S_{\mathrm{m}}$ where $R$ is the sensor resolution, we obtain a surface mass detection limit of

$$
L_{\mathrm{m}}=0.9 \mathrm{pg} / \mathrm{mm}^{2}
$$

\subsection{SABIO results discussion}

The detection limits achieved in SABIO, $5 \times 10^{-6}$ RIU and $0.9 \mathrm{pg} / \mathrm{mm}^{2}$, compares favorably to other published ring resonator results. Key to this was the use of multiple transducers on the chip to compensate for external disturbances, the high sensitivity of the slot waveguide ring resonators and the low system noise $1.2 \mathrm{pm}$, achieved by fitting an analytical model to the spectrum, thus effectively utilising all the information available. This is detailed in ${ }^{46}$. In terms of surface sensing, the detection limit of, presents a significant improvement of our own previously published value of $28 \mathrm{pg} / \mathrm{mm}$. As in the case of volume sensing, the improvement is mainly due to the reduced system noise.

\section{THE CONTINUATION IN INTOPSENS}

\subsection{InTopSens cartridges}

The InTopSens platform is also intended for use in a POC setting, and its packaged disposable part has been designed for non-critical alignment in a reader instrument with a fluidic channel network layer to transport the samples to the sensors. The integration of sample handling on-chip for the InTopSens device faces all of the same complexity as SABIO with regards to anti-clogging, removal/prevention of air bubbles and transport of assay fluids through microfluidics channels, pumps and valves. But while the SABIO design allowed 6 immunoassays, aiming for detection of biomarkers for liver 
cancer, hepatitis (B/C) or cytomegalovirus, InTopSens aims to test for some 64 bacterial DNA-strands in a fluidic blood sample for sepsis and to determine to which antibiotics these bacteria are resistant to. Here it is should also be noted that the InTopSens device includes on-chip amplification of DNA with polymerase chain reaction (PCR), so there is a tradeoff between assay time (number of PCR cycles) and minimum detection limit required of the photonic sensors.

The fluidic layer is on top of the optics whose light is coupled both in and out from above through alignment-tolerant grating couplers in a design that permits the simultaneous interrogation of multiple sensors. To minimize temperature influences, both active system control and on-chip references as listed in section 4.4 are used. The InTopSens temperature control has the added task of having to maintain a constant temperature during measurements, which occur in between PCR temperature ramping cycles.

\subsection{InTopSens transducer (design and fabrication)}

With a minimal footprint required per photonic transducer, one starting point for sensor development was a silicon-oninsulator (SOI) ring resonator, with a $70 \mathrm{~nm} / \mathrm{RIU}$ sensitivity for bulk changes of the refractive index, and a limit of detection of $10^{-5} \mathrm{RIU}$, which for biotin-avidin sensing corresponded to $10 \mathrm{ng} / \mathrm{ml}{ }^{21}$. In an aim to improve upon this detection limit whilst maintaining high integration, two approaches were taken. The first was based on a combination of experiments and simulations, and showed that a detection limit of $1 \mathrm{ng} / \mathrm{ml}$ is possible for the detection of avidin with the ring resonator if the measurement conditions are very well controlled, although this limits the practical POC applicability. A second approach was therefore taken to design sensors that can achieve lower detection limits in a robust way as outlined in the next subsection.

\subsection{InTopSens initial measurements and results}

In an aim to improve on limit of detection with the same high degree of integration as $i^{21}$, slot-waveguide racetrack resonators in the high-index-contrast SOI material system were developed ${ }^{30}$. The devices were fabricated using mass fabrication-compatible optical lithography, opening the way toward cheap, disposable chips. It was also proven that it is possible to apply surface chemistry inside a slot region that is only $100 \mathrm{~nm}$ wide. This is of importance for the application of nanophotonics for biosensing. A sensitivity of $298 \mathrm{~nm} / \mathrm{RIU}$ for bulk changes of the refractive index was recorded and from a calculated resonance wavelength resolution of $12.5 \mathrm{pm}$, the detection limit is $4.2 \times 10^{-5}$ RIU Due to the low quality factor of this resonator (330), principally due to bending and mismatch losses, this value is somewhat lower than that of the SABIO device.

\subsection{InTopSens future work}

Focus in InTopSens has therefore partially been shifted to other sensor designs. In the SOI material system with its high index contrast, Mach Zehnder interferometers can be scaled down and folded ${ }^{53}$, making it possible to integrate them in a dense sensor matrix. They are also robust against temperature and optical losses, and compatible with advanced waveguide technology (slot waveguides, slow light, etc). Furthermore the combination of interferometers with different interaction lengths enable extended dynamic range.

\section{ACKNOWLEDGEMENTS}

First we must thank Carl Fredrik Carlborg, who has contributed a lot of the work on microfluidics, packaging, and measurements summarized here. Other direct contributors to the SABIO work summarized here are Andrzej Kaźmierczak, Fabien Dortou, María José Bañuls Polo, Angel Maquieira Catala, Gerhard M. Kresbach, Thomas Moh, Laurent Vivien, Jon Popplewell, Gerry Ronan, Carlos A. Barrios, Göran Stemme and Wouter van der Wijngaart. Other direct contributors to the InTopSens work summarized here are Tom Claes, Jordi Gironès Molera, Katrien De Vos, Etienne Schacht, Roel Baets, and Peter Bienstman. As we review the collaborative projects SABIO and INTPOPSENS, many others have contributed. 
The SABIO project was financed by the European Commission through the sixth framework project FP6-IST-SABIO. The InTopSens project is financed by the European Commission through the seventh framework project FP7-ICTInTopSens. K. B. Gylfason acknowledges support of the Steinmaur Foundation, Liechtenstein.

\section{REFERENCES}

[1] Markov, D., D. Begari D., and Bornhop, D. J., "Breaking the $10^{-7}$ Barrier for RI Measurements in Nanoliter Volumes," Anal. Chem. 74, 5438-5441 (2002).

[2] Washburn, A. L., Gunn, L. C., and Bailey, R.C., "Label-free quantitation of a cancer biomarker in complex media using silicon photonic microring resonators," Anal. Chem. 81(22), 9499-9506 (2009).

[3] Sun, Y. S., Landry, J. P., Fei, Y. Y., Zhu, X. D., Luo, J. T., Wang, X.B., and Lam, K. S., "Effect of fluorescently labeling protein probes on kinetics of proteinligand reactions", Langmuir 24 (23), 13399-13405 (2008).

[4] De Feijter, J. A., Benjamins, J., and Veer, F. A., "Ellipsometry as a tool to study the adsorption behavior of synthetic and biopolymers at the air-water interface," Biopolymers 17(7), 1759-1772 (1978).

[5] Karlsson, R., Michaelsson, A., and Mattsson, L., "Kinetic analysis of monoclonal antibody-antigen interactions with a new biosensor based analytical system," J. Immunol. Methods 145, 229-240 (1991).

[6] Janasek, D, Franzke, J. and Manz, A., "Scaling and the design of miniaturized chemical-analysis systems," Nature 442, 374-380 (2006).

[7] Yariv, A., "Universal relations for coupling of optical power between microresonators and dielectric waveguides," Electronics Letters 36(4), 321-322 (2000).

[8] Gylfason, K. B., [Integrated Optical Slot-Waveguide Ring Resonator Sensor Arrays for Lab-on-Chip Applications], PhD Thesis TRITA-EE 2010:012, KTH-Royal institute of Technology, Stockholm, (2010).

[9] Tiefenthaler, K. and Lukosz, W., "Integrated optical switches and gas sensors," Opt. Lett. 9, 137-139 (1984)

[10] Lukosz, W. and Tiefenthaler, K., "Sensitivity of integrated optical grating and prism couplers as (bio)chemical sensors," Sensors and Actuators 15(3), 273-284 (1988).

[11] Tiefenthaler, K., and Lukosz, W., "Sensitivity of grating couplers as integrated optical chemical sensors," Journal of the Optical Society of America B: Opt. Phys. 6(2), 209-220 (1989).

[12] Lukosz, W. and Tiefenthaler, K., "Embossing technique for fabricating integrated optical components in hard inorganic waveguiding materials," Opt. Lett. 8(10), 537-539 (1983).

[13] Heideman, R. G., and Lambeck, P. V., "Remote opto-chemical sensing with extreme sensitivity: design, fabrication and performance of a pigtailed integrated optical phase-modulated Mach-Zehnder interferometer system," Sensors and Actuators B: Chemical 61(1-3), 100-127 (1999).

[14] Koster, T. and Paul Lambeck, P., "Fully integrated optical polarimeter," Sensors and Actuators B: Chemical 82(23), 213-226 (2002).

[15] Sohlström, H. and Öberg, "Refractive index measurement using integrated ring resonators," The 8th European Conference on Integrated Optics (ECIO '97), 322-325 (1997).

[16] Krioukov, E., Klunder, D. J. W., Driessen, A., Greve, J., and Otto. C., "Sensor based on an integrated optical microcavity," Opt. Lett. 27(7), 512-514 (2002).

[17] Chao, C. Y., and Guo, L. J., "Biochemical sensors based on polymer microrings with sharp asymmetrical resonance," Appl. Phys. Lett. 83(8), 1527-1529 (2003).

[18] Chao, C. Y., Fung, W., and Guo, L. J., "Polymer microring resonators for biochemical sensing applications," IEEE J. of Sel. Topics in Quantum Electronics 12(1), 134-142 (2006).

[19] Yalcin, A., Popat, K. C., Aldridge, J. C., Desai, T. A., Hryniewicz, J., Chbouki, N., Little, B. E., King O., Van, V., Chu, S., Gill, D., Anthes-Washburn, M., Unlu, M. S., and Goldberg, B. B., "Optical sensing of biomolecules using microring resonators," IEEE J. of Sel. Topics in Quantum Electronics 12(1), 148-155 (2006).

[20] Ksendzov, A., and Lin, Y., "Integrated optics ring-resonator sensors for protein detection," Opt. Lett. 30(24), 3344-3346 (2005).

[21] De Vos, K, Bartolozzi, I, Schacht, E., Bienstman, P., and Baets, R., "Silicon-on-insulator microring resonator for sensitive and label-free biosensing," Opt. Expr. 15(12), 7610-7615 (2007).

[22] De Vos, K., Girones, J., Popelka, S., Schacht, E., Baets, R., and Bienstman, P., "SOI optical microring resonator with poly(ethylene glycol) polymer brush for label-free biosensor applications," Biosensors and Bioelectronics 24(8), 2528-2533 (2009). 
[23] Almeida, V. R., Xu, Q., Barrios, C. A. and Lipson, M., "Guiding and confining light in void nanostructure,” Opt. Lett. 29(11), 1209-1211 (2004).

[24] Xu, Q., Almeida, V. R., Panepucci, R. R. and Lipson, M., "Experimental demonstration of guiding and confining light in nanometer-size low-refractive-index material," Opti. Lett. 29(14), 1626-1628 (2004).

[25] Carlborg, C. F., Gylfason, K. B., Kamierczak, A., Dortu, F., Bañuls Polo, M. J., Maquieira Catala, A., Kresbach, G. M., Sohlström, H., Moh, T., Vivien, L., Popplewell, J., Ronan, G., Barrios, C. A., Stemme, G. and van der Wijngaart, W., "A packaged optical slot-waveguide ring resonator sensor array for multiplex label-free assays in labs-on-chips," Lab Chip 10, 281-290 (2010).

[26] Barrios, C. A., Gylfason, K. B., Sánchez, B., Griol, A., Sohlström, H., Holgado, M. and Casquel, R., "Slotwaveguide biochemical sensor", Opt. Lett. 32(21), 3080-3082 (2007).

[27] Vivien, L., Marris-Morini, D., Griol, A., Gylfason, K. B., Hill, D., Alvarez, J., Sohlström, H., Hurtado, J., Bouville, D., and Cassan, E., "Vertical multiple-slot waveguide ring resonators in silicon nitride," Opt. Expr. 16(22), 1723717242 (2008).

[28] Ramachandran, A., Wang, S., Clarke, J., Ja, S., Goad, D., Wald, L., Flood, E., Knobbe, E., Hryniewicz, J., Chu, S., Gill, D., Chen, W., King, O. and Little, B., "A universal biosensing platform based on optical micro-ring resonators," Biosensors and Bioelectronics 23(7), 939-944 (2008).

[29] Hu, J., Carlie, N., Feng, N.-N., Petit, L., Agarwal, A., Richardson, K., and Kimerling, L., "Planar waveguidecoupled, high-index-contrast, high-q resonators in chalcogenide glass for sensing," Opt. Lett. 33(21), 2500-2502 (2008).

[30] Claes, T., Molera, J. G., De Vos, K., Schacht, E., Baets, R. and Bienstman, P., "Label-free biosensing with a slotwaveguide-based ring resonator in silicon on insulator,” IEEE Photonics J. 1(3), 197-204 (2009).

[31] Washburn, A. L., Gunn, L. C., and Bailey, R. C., "Label-free quantitation of a cancer biomarker in complex media using silicon photonic microring resonators", Anal. Chem. 81(22), 9499-9506 (2009).

[32] Washburn, A. L., Luchansky, M. S., Bowman, A. L., and Bailey, R. C., "Quantitative, label-free detection of five protein biomarkers using multiplexed arrays of silicon photonic microring resonators", Anal. Chem. 82(1), 69-72 (2010).

[33] F. Vollmer, S. Arnold, and D. Keng, "Single virus detection from the reactive shift of a whispering-gallery mode," Proc. of the National Academy of Sciences 105(52), 20701-20704 (2008).

[34] Armani, A. M., Kulkarni, R. P., Fraser, S. E., Flagan, R. C., and Vahala, K. J., "Label-free single molecule detection with optical microcavities", Science 317(5839), 783-787 (2007).

[35] S. Arnold, S. I. Shopova, and S. Holler, "Whispering gallery mode bio-sensor for label-free detection of singlemolecules: thermo-optic vs. reactive mechanism," Opt. Expr. 18(1), 281-287 (2010).

[36] Hanumegowda, N. M., Stica, C. J., Patel, B. C., White, I. and Fan, X., "Refractometric sensors based on microsphere resonators," Appl. Phys. Lett. 87(20), 201107 (2005).

[37] Fan, X., White, I. M., Zhu H., Suter, J. D. and Oveys, H., "Overview of novel integrated optical ring resonator bio/chemical sensors," Proc. SPIE 6452, 64520M (2007).

[38] Pfeifer, P., "Real time sensing of specific molecular binding using surface plasmon resonance spectroscopy," Sensors and Actuators B: Chemical 54(1-2), 166-175 (1999).

[39] Kabashin, A. V., Evans, P., Pastkovsky, S., Hendren, W., Wurtz, G. A., Atkinson, R., Pollard, R., Podolskiy, V. A. and Zayats, A. V., "Plasmonic nanorod metamaterials for biosensing," Nature Materials 8(11), 867-871 (2009).

[40] Fan, X., White, I. M., Shopova, S. I., Zhu, H., Suter, J. D., and Sun, Y., "Sensitive optical biosensors for unlabeled targets: A review," Analytica Chimica Acta 620(1-2), 8-26 (2008).

[41] Maire, G., Vivien, L., Sattler, G., Kazmierczak, A, Sanchez, B., Gylfason, K. B., Griol, A., Marris-Morini, D., Cassan, E., Giannone, D, Sohlström, H., and Hill, D., "High efficiency silicon nitride surface grating couplers," Opt. Expr. 16, 328-333 (2008).

[42] Kazmierczak, A., Dortu, F., Schrevens, O., Giannone, D., Vivien, L., Morini D. M., Bouville, D., Cassan, E, Gylfason, K. G., Sohlström, H., Sanchez, B., Griol, A., and Hill, D., "Light coupling and distribution for $\mathrm{Si}_{3} \mathrm{~N}_{4} / \mathrm{SiO}_{2}$ integrated multichannel single-mode sensing system," Opt. Eng. 48(1), 014401 (2009).

[43] Xia, Y. and Whitesides, G. M., Angew. "Soft Lithography," Angewandte Chemie International Edition 37(5), 550575 (1998).

[44] Duffy. D. C., Schueller. O. J., Brittain, S. T. and Whitesides G. M., "Rapid prototyping of microfluidic switches in poly(dimethyl siloxane) and their actuation by electro-osmotic flow," J. Micromech. Microeng. 9(3), 211-217 (1999). 
[45] J. C. Lotters, W. Olthuis, P. H. Veltink and P. Bergveld, "The mechanical properties of the rubber elastic polymer polydimethylsiloxane for sensor applications," J. Micromech. Microeng. 7, 145-147 (1997).

[46] Gylfason, K. G., Carlborg, C. F., Kazmierczak, A., Dortu, F., Sohlström, H., Vivien, L., Barrios, C. A., van der Wijngaart, W., and Stemme, G., "On-chip temperature compensation in an integrated slot-waveguide ring resonator refractive index sensor array", Opt. Expr. 18(4), 3226-3237 (2010).

[47] Harvey, A. H., Gallagher, J. S., and Levelt Sengers, J. M. H., "Revised Formulation for the Refractive Index of Water and Steam as a Function of Wavelength, Temperature and Density," Journal of Physical and Chemical Reference Data 27(4), 761-774 (1998).

[48] Takashashi, H., "Temperature stability of thin-film narrow-bandpass filters produced by ion-assisted deposition," Applied Optics 34(4), 667-675 (1995).

[49] Eldada, L., "Advances in telecom and datacom optical components," Opt. Eng. 40(7), 1165-1178 (2001).

[50] Suter, J. D., White, I. M., Zhu, H., and Fan, X., "Thermal characterization of liquid core optical ring resonator sensors," Appl. Opt. 46, 389-396 (2007).

[51] Lide, D. R. ed., [CRC handbook of chemistry and physics], CRC Taylor \& Francis distributor, (2008).

[52] Lee, J. N., Park, C. and Whitesides, G. M., "Solvent Compatibility of Poly(dimethylsiloxane)-Based Microfluidic Devices," Anal. Chem. 75, 6544-6554 (2003).

[53] Densmore A., Xu D. X., Janz S., Waldron P., Mischki T., Lopinsk G., Delâge A., Lapointe J., Cheben P., Lamontagne B., and Schmid J.H, "Spiral-path high-sensitivity silicon photonic wire molecular sensor with temperature-independent response," Opt. Lett. 33(6), 596-598 (2008). 